In the next place we have cases in which the uterus appears externally to be single, but the cavities have not coalesced, uterus septus. The partition may or may not be continued into the vagina.

Next come cases in which the uterus is only partly divided, having coalesced below but still with some divergence above. The divergence above may be considerable so as to give two distinct horns, uterus bicornis, or it may be no more than a slight dimpling in the middle line at the fundus so as to give the organ something of a heart shape, the uterus arcuatus. In these cases the division of the cavity is only partial.

Then, lastly, we have cases of what externally seems a single uterus, but with a partial septum, the uterus subseptus.

It may be added that these various forms may be somewhat complicated by one of the lateral organs being absent or rudimentary, so that we may have virtually a single horned uterus, or one in which only one half is fully developed.

I need not add anything as to the practical importance of remembering the existence of this malformation. I may only say that, had the practitioner who dealt with the case ever seen a specimen such as this, he would probably not have diagnosed extra-uterine pregnancy from the fact that he had found and emptied one uterus.

\title{
OBSERVATIONS ON THE SPINAL CORD
}

\section{IN THE INSANE.}

By R. S. STEWART, M.B. AND C.M.,

Senior Assistant Medical Officer, Glamorgan County Asylum, Bridgend.

Thesis for the Degree of M.D. in the University of Glasgow.

(Continued from page 101.)

THE next series of cases comprises six, in which the form of mental disorder was dementia.

CASE VI.-Summary-Delusions as to identity of persons and places-muttering to self-involuntary and unconscious passage of urine and alvine evacuations-paralysis of left external rectus-inequality of pupils, with loss of response to light and accommodation-absence of patellar reflexnormal superficial reflexes-common sensation not entirely obliterated - tactile sensibility present in some degree-intermittent clonic spasms of right triceps brachii-tremor of muscles of lips, and occasionally of muscles of abdomen and legs-ataxic gait and deficient equilibration increased by 
closure of the eyes-temporary monoplegia of right armarthropathy of right knee-eschars-coma-death.

Thomas S., a navvy, a native of Wiltshire, age 49, was admitted on 5th May, 1885. With regard to his antecedents very little information could be obtained. He had been in the workhouse for some time, and was on the "imbecile list" for fourteen months before his admission. The evidences of insanity given in his admission order were refusal of food, his saying he was in heaven, and running about naked.

On admission he was a stout well nourished man; height, 5 feet $7 \frac{1}{2}$ inches; weight, 11 stones 11 pounds. He was inclined to be communicative, and gave expression to some delusions, but shortly after admission he stubbornly refused to speak. He sat or lay in bed with his hands before his eyes, muttering semi-audibly to himself, and occasionally one could hear him repeat, "Curse me God, curse me God," or "take me up to heaven." He refused his food, giving, as his reasons for so doing, that he was in heaven, that he was "the happy angel," and that God would not allow him to take food. This refusal of food was persisted in, with few exceptions, up till the time of his death, and necessitated the regular administration, by the stomach tube, of food three times a day. There was internal strabismus from paralysis of the left external rectus; the left pupil was larger than the right, and both were irresponsive to light or accommodation. . The kneejerk was completely absent on both sides, but the plantar and other superficial reflexes were normal. There was almost constant dribbling of urine, of which, as well as of the passage of alvine evacuations, he appeared to be quite unconscious. The right arm was the seat of frequent, but not constant, tremor, having a rhythm and extent of movement somewhat similar to those of ankle clonus. This tremor was much intensified during the process of forcible feeding, and at such times it often extended to the abdominal muscles and legs. The tremor of the arm appeared to be due to clonic contractions of the triceps, and the intensification and radiation of the tremor appeared to be associated with the powerful contractions of the muscles of mastication made in his stubborn endeavours to resist the forcible opening of his jaws by the gag. There were also fine tremors of the muscles of the lips and levator menti. He felt as a painful impression the prick of a pin; he was able to recognise the contact of the head of the pin, to distinguish the head from the point, and to localise the point of contact pretty accurately. His gait was distinctly ataxic ; in standing or walking he kept his feet widely apart, and 
during locomotion the heels were brought down with the characteristic stamp. Equilibration, when the feet were in close apposition, was deficient, and the swaying was decidedly increased on closing the eyes. On 3rd June it was found that the right arm was completely paralysed; the limb hung helplessly by his side, and he was totally unable to execute the slightest movement. In three days there was a slight return of power, but the grasp of that hand continued feeble. On 10th June the right knee, which up till then was perfectly normal, was found in a condition which strongly suggested that it was a case of Charcot's joint affection. The left knee was normal. The right knee was considerably swollen, and presented the features of a hydarthrosis. The patella could be felt floating on the fluid contents of the articular cavity. But, in addition to the signs of articular effusion, there was very noticeable swelling and induration of the limb for some distance above and below the knee, so that there was considerable disparity between the measurements of the two limbs elsewhere than at the knee. This, as well as the variations in the degree of the swelling, is indicated in the accompanying table.

MEASUREMENTS OF LOWER EXTREMITIES.

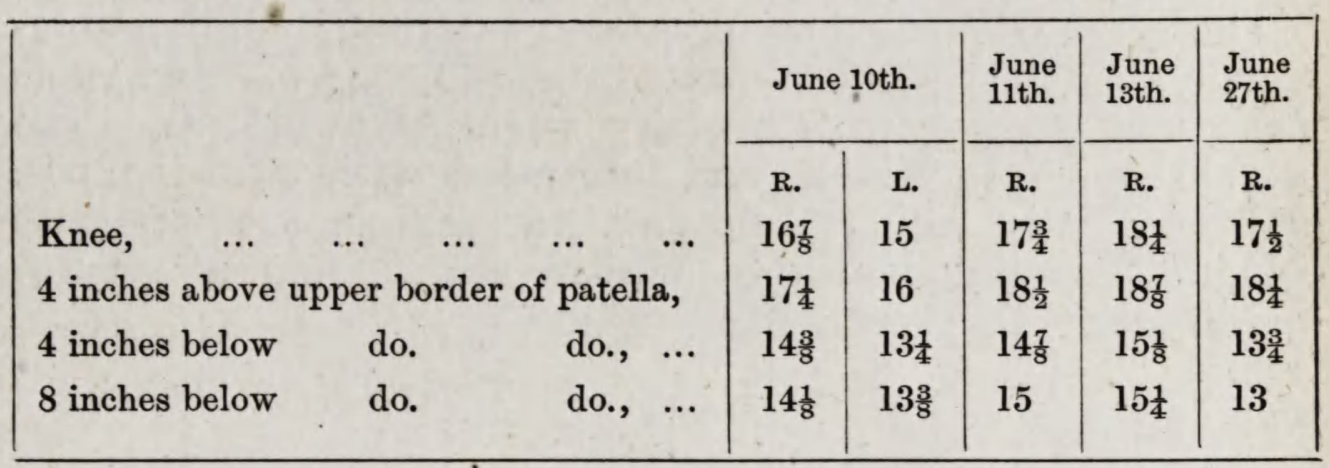

The movements of the joint, active and passive, were perfectly unrestricted, but were accompanied by a creaky feeling on placing the hand over the articulation. It appeared to give rise to no painful sensations: in fact, the patient was quite unaware of its existence until it was pointed out. $\mathrm{He}$ could move it freely, or allow it to be moved, and often he would walk energetically round the airing court without apparently suffering in the slightest degree. It appears to have been rather sudden in its onset, and no specific history of injury could be found. Two days after its first appearance, a slight ecchymosis was visible over the inner aspect of the head of the tibia, and next day, in addition to a further 
augmentation of the swelling, and the appearance of œdema of the foot and ankle, the ecchymosis had extended so as to involve the back of the thigh, over an area about the size of the hand, and the posterior and inner aspect of the calf. Two small bedsores which had formed shortly after admission, one over the sacrum, slightly to the left of the middle line, the other over the right gluteal region, now began to assume serious dimensions. That on the left penetrated as far as the sacrum, and presented a sloughy gangrenous mass of cellular tissue, tending to separate. During the next 14 days the swelling of the knee abated somewhat, but the eschars showed no sign of healing action. On 30th June he became comatose, the coma gradually deepened and ended in death, his residence being in all nearly two months.

At the autopsy the following changes were noted:-On the inner side of and above the knee a collection of sanguineous sero-purulent fluid was found, which was, however, perfectly extra-articular. On opening the joint, evidence of intraarticular changes were also to be noted; there was distinct erosion of the cartilage over the lower end of the femur, over an area about the size of a threepenny piece; the cartilaginous surface of the patella was roughened; there was no pus in the joint, but the synovia was slightly in excess.

The cerebro-spinal dura presented externally nothing noteworthy, but the soft membranes generally were highly hyperæmic and covered with a glairy purulent exudation. The sacrum was soft, friable, and infiltrated with offensive pus. The cord was rather soft, and, on section, the posterior columns, especially in the lumbar region, had a greyish appearance. Its measurements were-

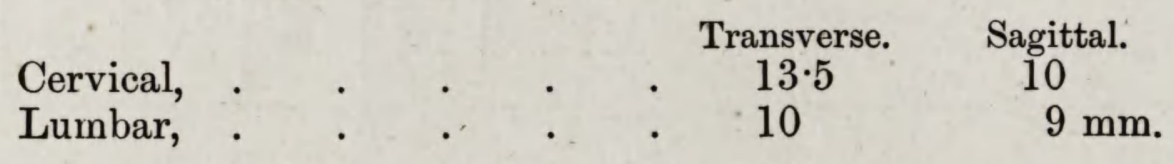

The brain weighed $43 \frac{1}{2}$ oz.; its tissue was soft and œdematous, but no gross lesion was discovered.

Microscopic examination.-The large pyramidal cells of the cerebral cortex are well defined both as regards outline and processes, but in many instances, in the carmine stained sections, they present traces of yellow granular degeneration. The degenerated parts of these cells are stained of a dark brown colour by osmic acid. Several of the arteries of the white matter are considerably distended, but this is by no means general.

Spinal Cord.-Changes are apparent throughout the whole 
length of the spinal cord, and these are limited almost entirely to two regions of the transverse section, the posterior white columns and the cornua of grey matter. Sections were stained with carmine, osmic acid, and by the hæmatoxylinprussiate method. In the cervical region the degeneration of the posterior columns is rendered apparent in the carmine tinted sections by the deeper colour which they assume. This degeneration exists over almost their whole extent transversely, varying, however, in intensity in different parts. The deeper parts-i.e., those lying next the grey commissure, are comparatively unaffected, while of the remaining parts of the columns, the degeneration is more pronounced in the internal divisions, and at the outer part of the external divisions, in which last position the sclerotic tissue appears to interrupt in some degree the fibres of the internal radicular fasciculus. (Fig. 2a.)

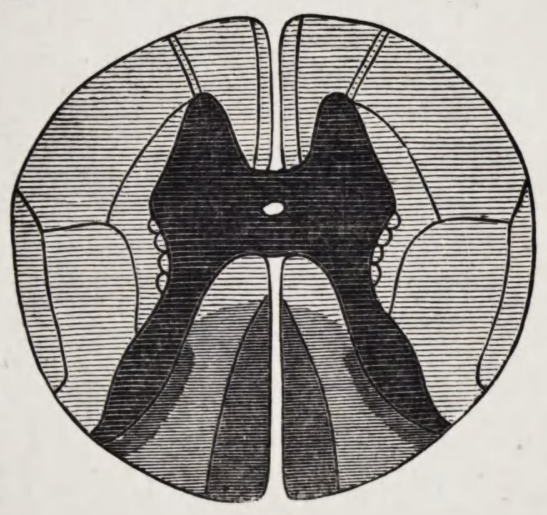

$2 \mathrm{~A}$

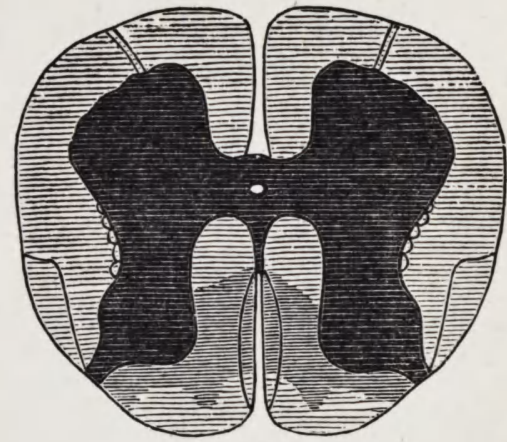

$2 \mathrm{~B}$

Fig. 2.- Spinal Cord from a case of dementia, with symptoms of locomotor ataxy. (a.) Cervical enlargement. The deeper parts of the posterior columns are unaffected, and the sclerosis is more pronounced in the columns of Goll, and in the outer part of the columns of Burdach. (b.) Lumbar enlargement,

In the lumbar cord the deeper parts of the posterior columns are again unaffected. In addition, however, the superficial parts of the columns of Goll, and, towards their inner side, of the columns of Burdach likewise are unaffected. The degenerated area extends transversely over the whole width of the posterior columns in their middle two-fourths, but posteriorly it is limited to the outer parts of the postero-external columns. (Fig. 2b.)

Both in the cervical and lumbar region there is a very apparent degeneration of the nerve cells of the central grey matter, and this applies alike to the larger multipolar ganglionic corpuscles of the anterior, and to the lesser cells of bipolar form of the posterior cornua. It exists to a pretty high degree; almost every one of the cells shows, in greater or less degree, 
evidences of degeneration, and many of them are most extensively degenerated. In a few instances there are, in addition to the degeneration, evidences of atrophy, but this is limited to only a few cells, and is by no means very pronounced. The majority of the cells are of normal size, and present well-defined outlines, processes, nuclei and nucleoli. The degenerated condition is remarkably well demonstrated by the use of osmic acid, which stains the granular parts of the cells of a dark brown colour. In the osmic acid stained sections, the degenerated areas of the white columns appear of a relatively pale colour. The nerve fibres of these areas are noticeably diminished both in number and size, while the glia cells are numerous and well developed. Corpora amylacea are not unfrequent over the degenerated areas, but they are also found in small number scattered generally over the section. In sections stained by Weigert's hæmatoxylin-prussiate method the degenerated condition of the nerve cells is well demonstrated, the granular parts appearing of a very dark colour; in the areas of columnar degeneration the connective tissue is stained of a light lavender brown, while the remaining myeline sheaths take on a dark blue colour. In all the sections it is observed that there is a distinct difference in the appearance of the new formation in different parts of the posterior columns; in the outer segments of the columns of Burdach, through which the internal radicular fasciculi pass, it has the character of well formed wavy fibrillar connective tissue, while in the remainder of the degenerated area it consists mainly of a thickening and coarseness of the normally fine connective tissue trabeculæ.

During life, this case presented many of the symptoms of locomotor ataxy, leading one to expect such a diseased condition of the posterior columns as was found on microscopic examination; but there were, in addition, other symptoms only to be explained by supposing an extension of the pathological process, to the central or trophic parts of the cord. It is in this way that the joint affection and the eschars are to be accounted for. The character of the symptoms manifested during life indicates an "irritative," as contra-distinguished from a "degenerative" lesion of the cerebro-spinal centre. This case affords an illustration of the view held by Erb, that sclerosis of the posterior columns is in the majority of cases, perhaps in all, not the only condition found in tabes. The process extends from the posterior columns to the lateral columns, the posterior cornua, and more rarely to the anterior * Ziemssen's Cyclopedia of Medicine, vol. xiii, p. 536. 
cornua, causing degeneration, sclerosis, and atrophy of the ganglion cells. The extension of the morbid process to the anterior cornua takes place along the fibres of the internal radicular fasciculus, which have been shown by Kölliker to be traceable to the anterior cornua, where they form connections with the ganglion cells. From the fact that, the joint affection was on the right side, and that the bedsores attained their greatest development on the left side of the middle line of the sacrum, it is probable that the morbid process in the central grey matter was of relatively greater intensity in the right half of the cord.

CASE VII.-Summary-Climacteric dementia of two years and five months' duration-premature senility-spontaneous development of eschars - defective sensibility - defective habits - increasing dementia and helplessness-coma-death.

Martha C., aged 53, a labourer's wife, a native of Somersetshire, was admitted on 9th March, 1885. The duration of the mental disorder was two years; it was characterised by a decided change of habits and character, delusions and mental failure. Previously a very respectable woman, she had fallen into drinking habits; became childish, silly, and emotional, unreasoning and incoherent. Her appearance was that of a prematurely old woman; she was grey-haired; and her memory, more particularly for recent erents, was much impaired. Habits on admission were not defective.

On 27th July ( $3 \frac{1}{2}$ months after admission), while her habits were still perfectly correct, and she was not confined to bed, but sitting or walking about by day, an eschar formed on each trochanteric region. At first this consisted of a slough of skin and cellular tissue about the size of a crown piece. Shortly afterwards she became more feeble and helpless, and more demented; her habits became defective, and she was confined to bed. The eschars became more and more grave, and another formed over the middle line of the sacrum. The patellar reflexes were noted at this time to be exaggerated. Death occurred on 12th August, about five months after admission, preceded for twenty-four hours by gradually deepening coma, lividity, and weak irregular pulse.

Autopsy.-The cerebro-spinal fluid was in considerable excess, and in it floated numerous cholesterine crystals. The brain was fairly firm; the soft membranes were nowhere adherent, but were gelatinous and opaque over the convexity; the central medullary substance presented a yellowish tinge, and the inner layer of the cortex was somewhat redder than 
normal. Over the pons there was a patch of recent meningeal hæmorrhage, about an inch in size (evidently the immediate cause of death). The basal ganglia were intact. The spinal dura mater presented nothing noteworthy; the soft membranes were injected inferiorly, and the cord itself was firm, and presented to the naked eye little appearance of alteration.

Microscopic examination-Brain.-There is an undue vascularity of the grey cortex, the small capillaries forming a prominent network. Hæmatoidin granules and crystals occur pretty frequently in the vascular sheaths, and here and there round cells are found collected round the vessels, some of them having in their interior pigment particles. As regards the nerve cells, the larger pyramidal cells of the third layer of the cortex appear ill defined. Their outlines are not well pronounced, and their processes are wasted looking. With the exception of the nucleus, they take on the carmine staining very imperfectly, presenting themselves as yellow granular bodies with a carmine tinted nucleus in their interior. The smaller pyramidal cells present traces, though to a much less extent, of the same yellow granular degenerative appearance.

Spinal Cord.-Sections were made of the cervical and lumbar enlargements, and of the dorsal region. In these

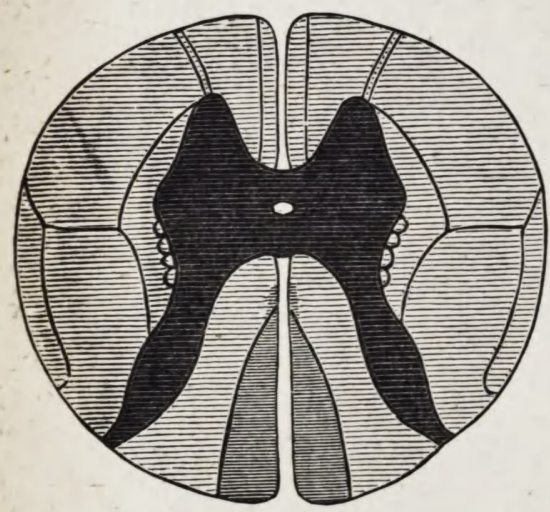

$3 \mathbf{A}$

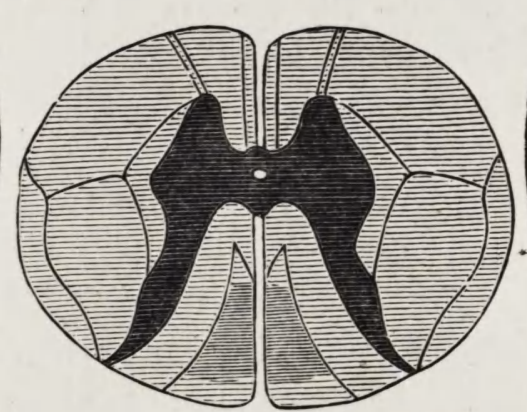

3 B.

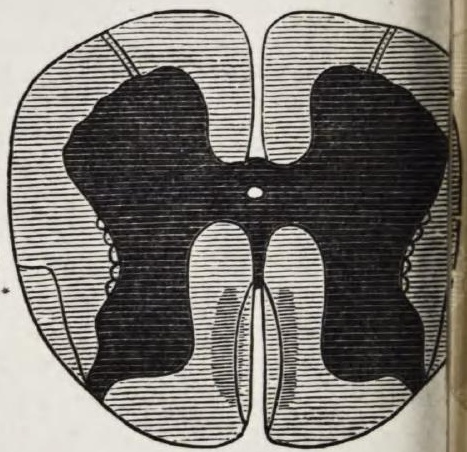

3 C.

Fig. 3.-Spinal cord from a case of climacteric dementia. Sclerosis of the postero-internal columns- $(a)$, cervical ; $(b)$, dorsal ; $(c)$, lumbar regions

regions evidences of grey degeneration of the columns of Goll are found. In the cervical region it is most distinct, and here it is strictly limited to the postero-internal columns, which, with the exception of a very small area at their deeper parts, take on with carmine a uniformly deeper tint than the other parts of the white substance. In sections stained by Weigert's hæmatoxylin-prussiate method, and with osmic acid, this area of degeneration appears as a lighter strip 
on each side of the posterior median fissure (Fig. 3a). In the dorsal region this degeneration is not so apparent, nor yet so definitely localised. It does however exist, affecting mostly the posterior part of each column, but not extending quite to the periphery (Fig. $3 b$ ).

It is likewise not so apparent in the lumbar region, where only a very small area of degeneration is to be observed, and that in an unequal degree on the two sides. It is best demonstrated in sections stained with osmic acid, where the degenerated areas appear as light patches in the midst of the dark normally stained columns. In one column the area of degeneration appears of an elongated oval form, while in the other it takes the form of a narrow strip running alongside the median fissure. In both columns the areas are completely surrounded with normal nerve tissue (Fig. 3c).

In this case I devoted considerable time and attention to the investigation of the condition of the nerve cells of the spinal cord and cerebral cortex. Firstly, sections of the lumbar cord (after hardening in the usual manner) were mounted unstained in glycerine. In these the nerve-cells, even with a low power $(\times 80)$, stand out prominently as yellow granular bodies in the light-coloured ground of the grey matter. They have somewhat badly defined edges and withered looking processes, and they show a tendency to assume the globular form. With a high power $(\times 300)$ it is seen that the majority of the large multipolar ganglion cells present a distinct yellow granular appearance. In some few cells this appearance is not presented, and here, as a rule, the nucleus and nucleolus are quite observable, but these latter, even in some of the apparently healthy cells, are occasionally rather obscured and indistinct. In many cases the granular condition is only partial in its distribution, in every case, however, appearing as a distinct cluster of granules occupying one or other of the angular recesses of the cell, and stretching inwards to the nucleus which appears at its inner margin. Higher grades of this degeneration are to be found, until finally it seems as if the whole cell were just one mass of granules. In these last cases two varieties are to be observed. All the advanced degenerated cells present this appearance in common, that they are of a distinct yellow colour, but in some it is easily observed that the nucleus is completely obscured, while in others that part is seen without difficulty. In the latter case the cells do not present the marked granularity of contents which is exhibited by those cells in which the nucleus is obscured. They appear to have more homogeneous, thou gh No. 3.

Vol. XXVI. 
still yellowish, contents. In those cells in which the nucleus is completely obscured, the contents are very granular, and the degeneration affects almost the whole cell, leaving often only a remnant of normal parenchyma at the periphery of the cell, or at one or other of their polar prolongations.

In sections soaked in dilute acetic acid, but little change is to be noted as regards those cells which are in a condition of advanced degeneration, and the only effect on the more or less healthy cell is an increased transparency of parenchyma and greater distinctness of the nucleus.

In sections treated with ether, after immersion for some time in alcohol, the following changes are observed:- There is a sensible clearing up of many of the cells, but this is in most cases only partial, while in not a few instances very little change is to be noted. This last remark applies to cells which are extensively degenerated, to those, namely, in which the whole cell-substance seems composed of a mass of granules, and in which the nucleus is quite obscured; but even in these there is some slight change in the direction of increased clearness compared with similarly degenerated cells not treated with ether. Examination of sections treated first by acetic acid and then by alcohol and ether gives practically the same results, as in the case of simple treatment by alcohol and ether alone.

In sections stained by osmic acid, the following appearances are noted:-The nerve cells are, almost without exception, stained in varying degrees of a dark brown colour. Even those cells which present no trace, or very little trace of granular appearance, are sensibly stained. It is in those cells which present the most advanced granularity that the stain is deepest, and in these the depth of staining varies in different parts of the cell. In some the granular mass is uniformly affected by the reagent, but in others a particular and often fairly defined part of the granular area is stained of a much deeper tint than the remainder. This deeper staining is usually of crescentic form, and appears to be related in some fashion with the nucleus.

In sections stained with diluted carmine fluid the nerve cells appear, even under a low power, as partly pink and partly yellow bodies, and under the high power the granular degeneration is very apparent. The degenerated area does not take on the staining, and so, in these cells which are most affected there is little or no appearance of carmine tinting.

In sections of the dorsal cord, the bipolar nerve-cells of the vesicular columns of Clarke are seen sharing in equal degree 
in the general degenerated condition, and in all the regions of the cord this degeneration affects, though in a less marked manner, the smaller cells of the posterior and anterior cornua, being by no means restricted to the cells of larger size.

The vessels in most of the sections appear to be considerably thickened from hypertrophy of their walls.

The condition of the cerebral cells was investigated in a similar manner to those of the spinal grey substance, with approximately similar results, although, in the case of the former, microscopical investigation is attended with considerably greater difficulties.

In this case the unsoundness of mind is to be looked upon as one, and practically the most prominent, of the symptoms of a wide-spread degenerative affection of the essential nervous elements, not of the higher or cerebral centres alone, but affecting alike the whole cerebro-spinal system. The morbid process, in all probability primarily of a "degenerative" kind, shows later on a tendency to take on an "irritative" character manifesting itself during life by the spontaneous occurrence of cutaneous eschars.

CASE VIII. Organic dementia-paralytic seizure on three separate occasions_right hemiplegia and aphasia.

Margaret J., aged 48, was admitted on 13th March, 1883. Four years before admission she had a paralytic stroke, causing right hemiplegia and aphasia. She recovered her speech, and six months prior to admission she had a second attack, which did not, however, affect her speech. The third seizure occurred 9 weeks before admission, and again involved speech. This was an ordinary case of right hemiplegia and aphasia, following a gross cerebral lesion, and accompanied by such a degree of mental disorder as required her detention in an asylum. During her residence she was often very restless, noisy, and emotional, frequently tossing about at night, getting out of bed, screaming and crying. She died on 17 th November, 1884.

At the autopsy very pronounced changes were found in the brain and some other organs. The cerebro-spinal fluid was in excess, and inside the cerebral dura mater there was a soft new membrane, apparently the result of chronic hæmorrhagic pachymeningitis. The soft meninges were gelatinous and opaque, and the subpial fluid increased. Two centres of softening were found, one on the left side corresponding to the middle of the ascending parietal convolution, the other on the right side situated about the middle of the external aspect of 
the occipital lobe. These softened areas extended from the cortex into the medullary centre, but did not reach the central ganglia which were unaffected.

The liver and kidneys were the seat of cystic disease, the latter weighing on the right 21 and on the left $19 \frac{1}{2}$ ozs. Nodules were found on the mitral curtains, and the lungs were affected with lobular inflammation and basal congestion. The measurements of the cord were-

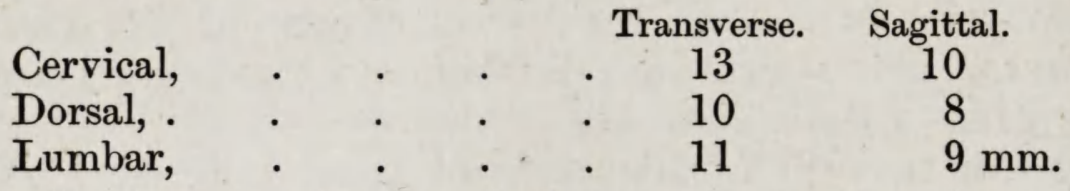

Methylated spirit was the hardening agent used, and sections were stained with carmine. These present the usual appearances of descending degeneration of the pyramidal tract secondary to a lesion in the left half of the brain. In the cervical region the right lateral column and the left column of Türck are stained of a deep colour. In the dorsal region the degeneration of the left anterior column does not exist, while in the lumbar region the degeneration is entirely limited to the crossed pyramidal tract, and here it is placed posteriorly, and reaches the periphery. The outline of the degenerated area in the lateral column throughout the cord is of a rounded form, and it has well defined borders, and is distinctly delimited from the surrounding nervous tissue. In the cervical region it does not reach so far forwards as the level of junction of the posterior columns and grey commissure, while in all three regions it is separated from the posterior cornua by a zone of healthy tissue.

CASE IX.-Summary-Acute mania-hereditary predisposition-gradual supervention of dementia-phthisis pulmonalis-emaciation and muscular atrophy - death.

George Henry S., a clerk, aged 27, was admitted on 22nd September, 1874, suffering from acute mania of three days' duration. No exciting cause could be assigned, but hereditary predisposition existed, his father and a sister having been insane. In the course of a month the excitement gradually abated, but he sank into dementia. This last continued and became deeper, and his habits became defective. Symptoms of phthisis developed, and he became emaciated. General muscular wasting. No bedsores. Death occurred on 19th October, 1885, the duration of the mental disorder being in all over eleven years.

Autopsy.-The spinal meninges were injected inferiorly, 
and the cord itself was soft and œedematous. Its weight was $19 \frac{1}{2}$ drams, and its measurements-

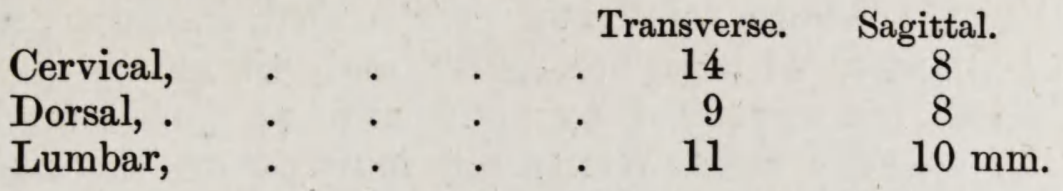

The calvarium was thickened over the centre of the occipital bone, and weighed 13 ozs. The cerebral soft membranes were gelatinous and lactescent over the convexity, but nowhere adherent. The brain weighed 56 ozs., and the hemispheres each $24 \frac{1}{2}$; it was soft and œdematous, and the arachnoid fluid was in excess. The convolutions and, on section, the cortical grey substance appeared wasted. The lungs were affected with advanced tubereular disease, and the liver was fatty.

Microscopic Examination.-Sections of brain and spinal cord were examined unstained and stained with carmine and osmic acid. The large pyramidal cells of the cortex are everywhere considerably degenerated and atrophied; their outlines are ill defined and irregular; their processes wasted, and the whole cell presents a granular yellow appearance. In the osmic acid stained sections these cells present a dark brown appearance. No very marked changes are to be observed in the blood-vessels. The neuroglia is coarse. In unstained sections of the spinal cord, the cells of the anterior cornua are seen to be in an advanced state of degeneration. They are partially, in a few instances even wholly, occupied by yellow granular contents which, in many cases, quite obscures the nucleus. These cells, with osmic acid, take on a decidedly dark brown tint. There is likewise a degree of atrophy, many of the cells being ill defined, with rounded angles and badly marked processes. A few of the vessels of the grey are unduly dilated, but no alteration of white substance is to be observed.

CASE X.-Dementia-second attack of insanity-history of apoplectic seizures-hereditary predisposition-habitual alcoholic intemperance - cardiac disease-diminished voluntary motor power-aggravation of cardiac condition-death.

David M. age 51, a labourer, was admitted on 5th May, 1884, suffering from his second attack of mental disorder of four days' duration. He is said to have had a fit twelve months, and a second one shortly, before admission. An aunt and her mother suffered from insanity. His general character and habits were essentially bad; he was a habitual drunkard, and 
ill-treated his wife. On admission he was a man of medium height, well nourished, with fairish hair, and injected facial capillaries. There was very pronounced cardiac, aortic and mitral disease, with hypertrophy and dilatation of the left ventricle. He appeared to be somewhat feeble on his legs, and the patellar reflex was more marked on the right side. There was very noticeable tremor of the head and neck, and enfeebled grasping power.

His mental condition was one of mild dementia; he seemed stupid and quite indifferent about his coming to the asylum, sat spitting about carelessly on the furniture or any one near, and his appearance generally was untidy. His general condition during his residence was one of drowsy, somnolent stupidity; he was extremely disinclined to do any work; he sat by the fire all day; taking little notice of his surroundings, and often falling asleep. Ten months after admission he had what was described as an "epileptic fit," and 17 months after admission the cardiac condition became aggravated, and he died after a residence of about 18 months.

The autopsy was performed 38 hours after death. In addition to grave changes in the other organs, the following were noted as regards the cerebro-spinal axis. There was considerable excess of cerebro-spinal fluid. The spinal cord weighed $21 \frac{1}{2}$ drams; it was soft and œedematous, and its measurements were-

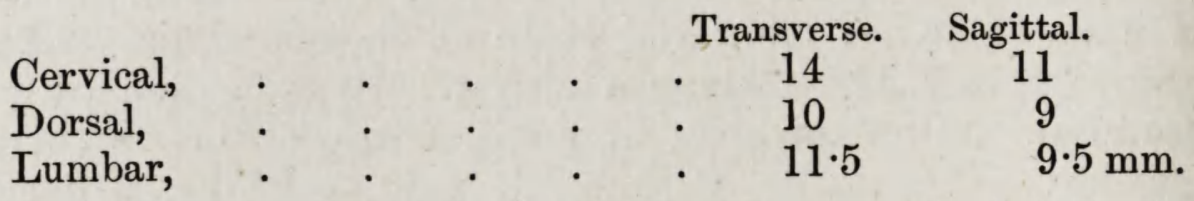

The dura and pia spinalis were injected, especially inferiorly. The calvarium weighed $14 \frac{1}{2}$ ozs., and was adherent to the dura. The brain weighed 48 ozs.; the soft membranes were gelatinous and opaque, but not adherent, being separated from the underlying convolutions by a considerable quantity of subpial serosity. The brain tissue was soft and odematous, and the central white substance was slightly brownish. The second frontal convolution on the right side was in great part replaced by a brownish gelatinous material extending inwards as far as an inch from the longitudinal sulcus. This was apparently the result of softening from embolism.

Microscopic Examination-Brain.-The larger and medium sized pyramidal nerve cells of the cortex present a degree of yellow granular degeneration, and in addition there is in the former a slight atrophic tendency. In no case, however, does the degeneration or atrophy proceed to such an 
extent as to obscure the nucleus. The remaining cells present no noticeable change. The cortex is unduly vascular, and the smaller arteries have slightly thickened walls. The neuroglia is cloudy and granular-looking.

In the spinal cord changes are apparent in the nerve cells, the blood-vessels, and the neuroglia. The ganglionic corpuscles of the anterior cornua, both in the cervical and lumbar enlargements, present a pronounced degree of degeneration. In unstained sections this degeneration is apparent even with a low power, varying in degree in different cells; in some confined to one or several of the angular recesses, and in others filling more or less the whole cell till, finally, the nucleus is completely hidden. The degenerated parts of the cells are of a granular appearance and yellow colour. With ether this is somewhat cleared up, the whole cell appearing more transparent, without, however, any material change of the colour of the granular parts. In sections stained with osmic acid, the degenerated parts of the cells take on a decidedly deeper tint, in some almost approaching a black colour. In carnine-tinted sections the granular parts of the cells not taking on the staining appear of a bright yellow colour, the remainder of the cell assuming the usual tint. In these last sections the degeneration is most apparent. The smaller arteries of the central grey substance have much thickened walls, and in many instances the perivascular space is distended and occupied by a homogeneous, partly fibrillar material stained very faintly with carmine. This is most apparent in the arteries of the anterior commissure, but it affects the others likewise, though in less degree. The increased thickness appears to depend mainly on a hypertrophy of the circular muscular coat. The neuroglia generally is swollen-looking, clouded and granular, but otherwise no change is to be found in the white substance.

With regard to the etiology of this case, three separate factors are to be recognised-hereditary predisposition, cardiac disease, and habitual intemperance. These two last, acting upon a brain hereditarily unstable, brought about a condition of degeneration of the essential nervous elements, a condition which manifested itself during life by a general failure of the mental faculties. The degeneration is, however, not confined to the highest nervous centres alone; it affects simultaneously and in almost equal degree the lower division of the nervous axis. The similarity of the pathological alterations, the degenerative atrophy of the larger pyramidal cerebral, and of the multipolar ganglion spinal cells, the vascular 
changes and alteration of neuroglia in the two divisions of the cerebro-spinal axis, forms a striking feature of this case.

CASE XI.-Head injury-epilepsy-paralytic seizuredementia-urethral stricture and retention-general analgesia-increase of patellar reflex-deficiency of superficial reflexes-uncertainty of gait-transitory attacks of maniaunilateral clonic convulsions-coma-death.

William D. N., an ironmoulder, aged 38, was admitted on 24th December, 1885, and died on 26th January, 1886. His father was a habitually intemperate man; one sister died of consumption, and a brother of "sunstroke." He himself was always a temperate man. Twenty-five years before admission he sustained a severe injury to his head, and 14 years afterwards "fits" supervened. Nine years before admission he had a "paralytic stroke" which affected his left side, but from which he completely recovered. For two years prior to his admission his mind had been noticeably weakened, and he had not been able to follow his occupation. The fits did not occur very frequently, perhaps to the number of two or three in a year, but they were described as being very severe, especially so during the two years before his admission. The last fit occurred three weeks before admission, and was followed by loss of consciousness which lasted for five days, during which he took no food, while bedsores formed.

Of late a difficulty of swallowing, and a tendency to choke while eating, were observed, and his sleep had become very indifferent.

On admission he was a tall, spare man, with dark hair, sallow anæmic complexion, and blue irides. His bodily condition was much below par- $\frac{\text { height }}{\text { weight }}=\frac{5 \mathrm{ft} .9 \frac{1}{2} \mathrm{in} .}{10 \mathrm{st.9}} \mathrm{lbs}$. Two eschars, unhealthy looking, with sloughy edges, and no sign of healing action, existed over the glnteal region, that on the right being the larger. His expression was dull, stupid, and vacant. Tongue clean and moist. Heart's action weak and irregular, both in rhythm and force; no cardiac murmurs. There was a slight urethral stricture, about half an inch, behind the external orifice, and retention of urine dependent partly on the stricture, but in part also on a paralytic condition of the detrusor, rendering catheterism necessary. The pupils were equal and contracted, dilating only very slightly on the withdrawal of light. There was complete analgesia of the trunk, limbs, and head. No pain was experienced when a fold of skin was transfixed, the sensation being merely one of touch. 
The sense of contact, localisation, and the distinction of objects, such as the head from the point of a pin, were retained in some degree. The patellar reflex was in slight excess, more so on the left side. The plantar reflex was normal, but the other superficial reflexes were in abeyance. There was slight rigidity of both legs; his gait was uncertain and unsteady, without any real loss of equilibration, even when the eyes were closed, and grasping power was decidedly impaired.

His mental condition was one of considerable hebetude; he was stupid and obtuse, and manifested great confusion of ideas as to time, places, the value of pieces of money, \&c. His memory was much impaired both for recent and past events.

During the first week of his residence catheterism was repeatedly required. There was obstinate constipation, and his habits were defective. During the succeeding week transient attacks of mania occurred, and twenty-six days after admission clonic convulsions supervened. At first these were limited to the left side, affecting the arm, leg, and face, with conjugate deviation of the eyes, and rotation of the head to the left. On the first day only three occurred, but gradually they became more frequent and more severe, so that on the third day consciousness was completely abolished in the intervals. During the intervals the head and eyes were turned to the right, and the left limbs were rigid. The knee jerk became more exaggerated on both sides, and clonus could be induced with facility by downward pressure of the patella. There was no ankle clonus, and the superficial reflexes remained as before. The clonic seizures lasted in all eight days, the number being 441. Although at first limited to, and always commencing in, the left side, latterly they showed a tendency to invade the right side. During the last three days of his life the average daily temperature was $101^{\circ} \mathrm{F}$.; pulse, 130 ; respiration, 36 per minute. Treatment was by chloral and bromide of potassium, nutritive enemata, and evaporating lotion to the head. The obliteration of consciousness became more and more pronounced, passing into coma, which gradually deepened, and ended in death.

The autopsy was performed thirty-three hours after death. The cerebro-spinal fluid was in excess. The spinal dura presented nothing. noteworthy; the soft membranes were congested; the cord weighed 19 drams, and its measurements were-

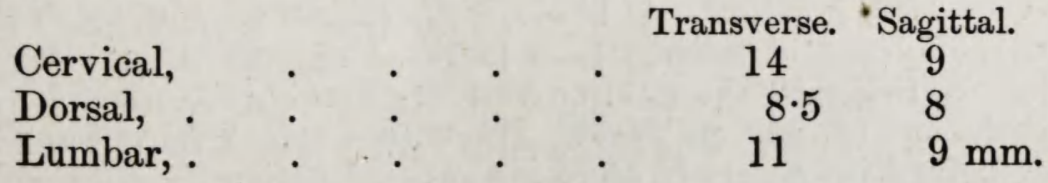


On section the changes apparent to the naked eye were increased vascularity of the central grey substance, and, probably, a slight greyish discoloration of the left lateral column in the cervical region. The calvarium weighed 14 ozs.; the dura presented no alteration; the brain weighed $47 \frac{1}{2}$ ozs. ; the right hemisphere 18; the left $19 \frac{1}{2}$; cerebellum and medulla $6 \frac{1}{2}$. The soft membranes generally were congested, and in addition were, over the convexity, slightly opaque and gelatinous. The congestion was distinctly more marked over the right hemisphere. The basal arteries were normal. The orbital surface of both frontal lobes had a shrivelled, wasted appearance and rusty brown colour, while the olfactory bulbs were extremely atrophied. There was adhesion of the soft membranes and decortication of the tips of the underlying convolutions over an area on the right side, corresponding to the upper $1 \frac{1}{2}$ inches of the ascending parietal convolution, and over the anterior part of the superior parietal lobule for about a superficial inch.

Elsewhere they were not adherent, not even over the orbital surface, where the soft membranes were separated from the cortex by a small quantity of brownish fluid. The brain tissue generally was soft and odematous; on section the cortex appeared extremely congested, more so on the right side; the puncta cruenta were well marked; the ventricular fluid was in considerable excess, and the lining membrane of the lateral and fourth ventricles presented numerous granulations. The cortex over the area of meningeal adhesion was relatively atrophied, while that of the orbital convolutions was considerably disorganised. Elsewhere it was not notably atrophied, only of a dark purple colour. Basal ganglia congested, more so on the right; otherwise unchanged.

Pieces of the right cortex, the right crus, medulla oblongata, and cervical and lumbar cord were hardened in Erlicky's fluid.* The left cortex, and crus, and pieces of cord were hardened in the usual manner in Müller's fluid. Sections were examined unstained, stained in Müller's fluid, with carmine and osmic acid, and with these last two combined.

In sections of the convolutions of the right side, taken from the seat of meningeal adhesion, the following appearances are

* This hardening agent is composed of sulphate of copper, 0.5 grams; bichromate of potassium, 2.5 grams; water, 100 grams (Archives de Neurologie, vol; x, p. 52). It hardens the tissues very rapidly; but, unless considerable care is taken, there is great risk of their being rendered brittle. In this case the solution was changed on the first and third day and at the end of the first week. The tissues were removed in a fortnight, and even then they were if anything rather brittle. 
noted:-There is very intense vascularity of both grey and white substance, most pronounced in the deeper layers of the cortex; the capillaries and small arteries are gorged with blood and stand out prominently. In places the arterial walls are somewhat thickened, and hæmatoidin deposits occur with some frequency in the perivascular lymphatic sheaths. With a view to examine for granule cells,* sections were immersed for some time in Müller's fluid, and others were stained with osmic acid. These granule cells are exceptional in their occurrence, only a very few being observed in the medullary centre of the convolution. In carmine tinted sections the larger pyramidal cells of the third layer of the cortex are observed to be degenerated and atrophied, presenting themselves as partly yellow and partly pink bodies with indistinct outlines, and a tendency to wasting of processes. The superficial cortical layer, that devoid, or nearly so, of nerve-cells is spongy and swollen-looking, and has numerous prominent neuroglia cells. In the sections of the corresponding convolution (ascending parietal) of the left side, there are two apparent differences. The large pyramidal cells, though presenting a degree of granular degeneration, do not show much change in the direction of atrophy. They are large and well-formed, with distinct outlines and well-marked processes. The increased vascularity is noticeably less than on the right side.

Crura Cerebri.-Sections were examined unstained and stained, with osmic acid, picro-carmine, and carmine. There is no material difference in the crura of the two sides; both are unduly vascular, and the nerve cells of the locus niger are in equal degree affected with granular degeneration. Only exceptionally do granule cells occur, and there is no appreciable appearance of a process of sclerosis either in the region of the pyramidal tract, or elsewhere; certainly none at all comparable to that which will afterwards be described in the case of the spinal cord.

Medulla Oblongata.-Sections were stained as in the case of the crura, and here again no process of sclerosis is to be detected either in the anterior pyramids, or elsewhere. The

* The methods used by Charcot (Archives de Neur., x, 31) for the investigation of granule cells are as follows:-Sections are made at the autopsy and immersed in Müller's fluid, examined immediately or after remaining for some hours or days in the liquid. Where tissues have been hardened in Muiller's fluid, sections are submitted to the action of osmic acid, which stains the granule cells of a much deeper black than the myeline sheaths among which they lie. Weigert's hæmatoxylin-prussiate method does not give such good results. 
only apparent alterations are the increased vascularity, and the degeneration of the nerve cells of the grey substance.

Spinal Cord.-With regard to the white substance, changes are apparent even to the naked eye. In carmine stained sections areas of deeper staining, indicating a process of degeneration, are observed in the lateral columns and the inner divisions of the posterior columns. In the case of the lateral columns, the degenerative process appears to be more intense on the left side, as indicated by a deeper staining. In the cervical region the degenerated area is of a triangular form; its anterior margin, rather indistinct, and shading off into the normal tissue in front, is on a level with the central canal; internally, it is in contact with the posterior cornu; and externally, it is separated from the periphery by a narrow zone of normal tissue. In the dorsal region this area is smaller,

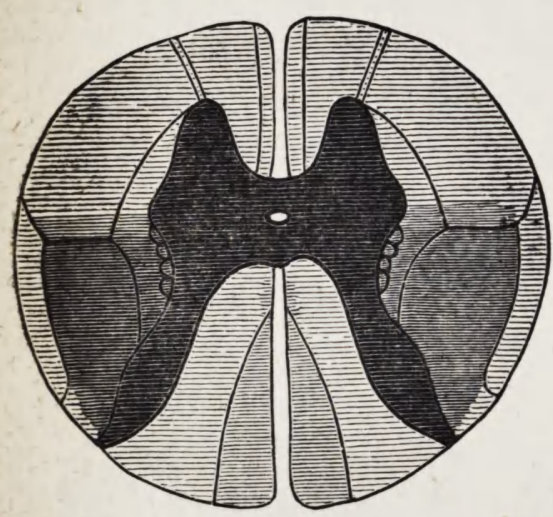

4 A.

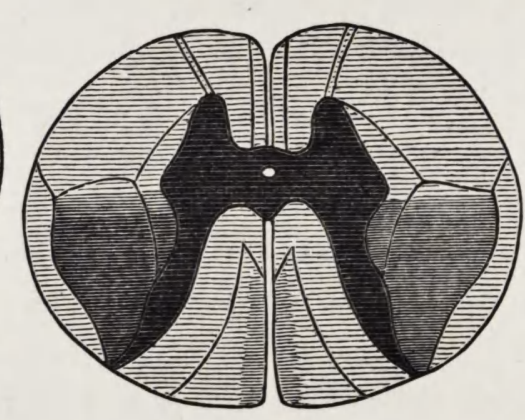

$4 \mathrm{~B}$

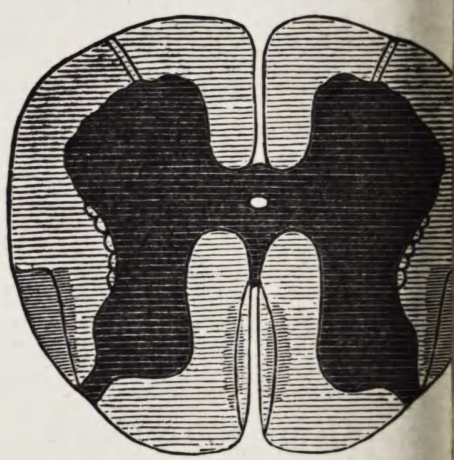

4 C.

Fig. 4.-Spinal cord in a case of dementia with epilepsy-degeneration of lateral columns and of postero-internal columns. (a.) Cervical. (b.) Dorsal. (c.) Lumbar regions.

but occupies much the same position, while in the lumbar region its size is much diminished, and here it is placed more posteriorly; is in contact with the pia mater, and is separated from the posterior cornu by a strip of normal tissue.

The degeneration of the posterior columns is not so pronounced as in the case of the lateral columns, and it is strictly confined to their inner division. In the cervical region it forms a wedge shaped area, reaching from the periphery nearly to the central grey matter; in the dorsal region it has diminished to a small strip on each side of the posterior median fissure, while in the lumbar enlargement it forms a small oval area lying embedded within normal tissue.

These areas present all the characters of sclerosis, increase of the supporting connective tissue elements, diminution in number and size of the nerve tubes, increased vascularity, and thickening of the arterial walls. 
In sections stained with carmine and osmic acid, a few granule cells are observed in the degenerated areas, and these last are very definitely mapped out by this combined staining, appearing as lighter pink tinted areas in the midst of the dark osmic-acid-stained medullary tissue. There is a very noticeable increase of vascularity of the whole transverse section, both white and grey, but it is most pronounced in the area of degeneration of the lateral columns.

The nerve cells of the central grey substance do not appear to be much atrophied; they have well defined edges and processes, but they present in varying degrees traces of yellow granular degeneration. This is very apparent even in unstained sections, but is admirably demonstrated in the sections stained with osmic acid and carmine. In these the cells appear as partly pink and partly dark brown bodies, the degenerated part being stained with the osmic acid, the remainder taking on the usual carmine tint. This applies most to the multipolar cells of the anterior cornua, but it likewise exists, though in minor degree, in the smaller cells of the posterior cornua and those of the vesicular columns of Clarke.

The central canal is obliterated.

The starting point of the pathological alteration, in all probability, is the disorganisation of the cortex of the orbital convolutions, a condition probably induced by the head injury which occurred twenty-five years before the death of the patient. This determines a morbid process, of an irritative type, which shows a tendency to extend in a progressive, though unequal fashion, being more intense in some parts than others. Epilepsy and dementia are secondarily induced. The morbid process attaining a greater intensity, as evidenced by the meningeal adhesions, in the region of the ascending parietal convolution and superior parietal lobule of the right side, determines a transient paralysis of the left side, and, the irritative quality of the lesion becoming pronounced, the unilateral clonic spasms which were ultimately the immediate cause of death. The majority of the symptoms are indicative of a diseased condition of the higher division of the nervous axis, but there are others which point to an implication of the lower or spinal division, such as the increase of the patellar reflex, the rigidity of the lower limbs, the bedsores, and the paralysis of the detrusor urinæ.

(To be continued.) 